\title{
Impairment of remote memory after closed head injury
}

\author{
HARVEY S LEVIN, ${ }^{*}$ WALTER M HIGH, ${ }^{*}$ CHRISTINA A MEYERS, $\dagger$ \\ ALEXANDER VON LAUFEN, * MARY ELLEN HAYDEN, $\dagger$ HOWARD M EISENBERG*
}

From the Division of Neurosurgery, ${ }^{*}$ The University of Texas Medical Branch, Galveston, and Department of Rehabilitation Medicine, $\uparrow$ Plaza Del Oro Medical Center, Houston, Texas, USA

SUMMARY Evidence of partial retrograde amnesia for episodic memories of no personal salience was found in head injured patients $(n=10)$ tested during posttraumatic amnesia or shortly after its resolution $(n=10)$, but there was no selective preservation of the earliest memories. In contrast, head injured patients tested during posttraumatic amnesia exhibited relatively preserved retention of early autobiographical memories which they recalled as accurately as oriented head injured patients. It is suggested that reminiscence of salient, early events increases their resistance to partial retrograde amnesia and contributes to the observed temporal gradient.

Case reports published during the 19th century documented the retrograde loss of memory for events shortly before a non-missile head injury while retention of very remote information was relatively spared.' In 1882 Ribot $^{2}$ inferred from these reports that memories become increasingly resistant to disruption as a function of their age and repetition (for example, rehearsal). Consistent with Ribot's postulation was Russell's finding that the interval of retrograde memory loss, which his patients estimated retrospectively, was relatively brief $(\leqslant 30$ minutes) in $86 \%$ of consecutive admissions for non-missile head injury as compared to the duration of anterograde amnesia which persisted beyond 24 hours in $40 \%$ of the cases. ${ }^{3}$

In some head injured patients retrograde amnesia (RA) initially extends to events several years in the past and gradually resolves beginning with the oldest events until a point is reached contiguous in time to the injury which remains as a gap in memory. ${ }^{4-6}$ Benson and Geschwind ${ }^{4}$ attributed this "shrinking" RA to recovery of memory retrieval because encoding and storage of events which occurred in the distant past are presumably completed before the injury. In contrast to Russell's reports concerning the interval of complete RA, Williams and Zangwill ${ }^{7}$

Address for reprint requests: Harvey S Levin, Ph.D. Division of Neurosurgery, The University of Texas Medical Branch, Galveston, Texas 77550, USA.

Received 6 July 1984 and in final revised form 14 October 1984. Accepted 29 October 1984 found evidence of partial retrograde memory loss for details of more remote events when they interviewed head injured patients after resolution of posttraumatic amnesia (PTA).

Recently developed standardised techniques permit assessment of varying degrees of retrograde memory loss for events which occurred in the public domain over a long interval. Squire, Slater and Chace ${ }^{8}$ found that a course of bilateral electroconvulsive therapy in depressed patients disrupted memories acquired within three years before treatment while sparing older memories.

Although investigators of amnesic disorders secondary to bilateral mesial temporal lobe removal, ${ }^{4}$ diencephalic injury, ${ }^{10}$ or alcoholic Korsakoff's syndrome $^{1112}$ have confirmed a similar temporal gradient of remote memory deficit using titles of television programmes, public events, and faces of prominent persons, the insidious or prior onset of memory disorder in the patients studied implies that their apparent RA may reflect progressive impairment in learning and retention of events (anterograde amnesia) rather than a retrograde loss of previously acquired memories. Further, several investigators have found no evidence for sparing of very remote memory in amnesic patients ${ }^{1314}$ and healthy elderly subjects. ${ }^{15} 16$

To define precisely the onset and temporal extent of RA for episodic events and personally salient information, we prospectively studied patients during the early stages of recovery from severe or moderate closed head injury. By confining the study to 
patients with no antecedent history of neuropsychiatric disorder and with satisfactory scholastic achievement, we assumed that their preinjury memory functioning was normal. In Study I we tested the hypotheses that (1) a quantitative test of episodic memory for events of no personal salience could demonstrate RA during the early stages of recovery after closed head injury; (2) the severity of RA for episodic memory is greater in head injured patients examined during PTA as compared to patients who have emerged from PTA; and (3) Ribot's formulation that memory for events from the remote past is more resistant to disruption than retention of relatively recent events irrespective of the class or type of memory. ${ }^{2}$ To test the second and third hypotheses using salient, personal memories, we employed an autobiographical memory technique in Study II.

\section{STUDY I}

\section{Patients and methods}

\section{Patients}

Two groups of 10 closed head injury patients studied during the course of inpatient rehabilitation after transfer from the neorosurgery service of one of the three university hospitals in the Houston-Galveston Comprehensive Central Nervous System Injury Trauma Center. The first closed head injury group was tested during PTA, which we assessed daily by a brief, bedside examination ${ }^{17}$ or orientation to person, place, time, recall of circumstances related to the hospitalisation, events before and after the injury. The second closed head injury group was normally oriented and no longer in PTA when they were given the remote memory test. Data supporting the reliability of scoring this schedule of questions and depicting the normal range have been reported. ${ }^{17}$ Apart from the criterion of PTA, we selected consecutive head injured patients to obtain two groups who were closely matched on age, education, and severity of initial injury as reflected by the Glasgow Coma Scale (GCS) ${ }^{18}$ and the interval during which the patient failed to follow simple commands (table 1). Other selection criteria included a preinjury history free of neuropsychiatric disorder, including no previous head injury, learning disability, or chronic substance abuse. We excluded aphasic closed head injury patients because of the verbal requirements of the tests.

Severe closed head injury (GCS score $\leqslant 8$ ) predominated in both the PTA ( 9 of 10 patients) and post-PTA (all 10 patients) groups. A single patient in the PTA group had an initial GCS score of 11 which was compatible with a nonmissile head injury of moderate severity. Table 1 shows that there were no significant differences between the patient groups in age, education, or initial GCS scores. According to computed tomographic (CT) and surgical findings, the PTA group included nine patients with hemispheric mass lesions (two left hemisphere, three right, four bilateral) and a single case of diffuse injury, whereas the oriented group tested after resolution of PTA included seven patients with hemispheric mass lesions (three left, one right, three bilateral) and three cases of diffuse injury. We also administered the remote memory test to 187 undergraduate students at the University of Houston to obtain normative data. Although the educational level of the college students surpassed that of the head injured groups, pilot testing of normal subjects in our laboratory disclosed no relationship between education and performance on this test.

\section{Procedure}

We revised and extended the remote memory test for titles of television programmes developed by Squire and Slater ${ }^{19}$ which asks the subject to select from four choices the title of a weekly programme which had been broadcast during evenings on a major network for a single season. This technique reflects exposure to television viewing and is sensitive to remote memory deficit. ${ }^{101920}$ There were six test items for each of five time periods: 1968-1971, 1972-1975, 1975-1977, 1977-1979, and 1980-1981. We verified that the target programmes were broadcast in the Houston vicinity and other Texas cities during the designated season and that the patients and control subjects were television viewers who had resided in Texas throughout the 1968-1981 period. All testing was completed during the fall and winter of 1981-1982, that is, after cancellation of the 1981 programmes. To facilitate data acquisition from patients during relatively early stages of recovery from head injury, we administered one-third (10 items) of

Table 1 Demographic and clinical features of head injured and control groups given the 1968-1981 Remote Memory Test of Television Programmes*

\begin{tabular}{|c|c|c|c|c|c|c|}
\hline & \multicolumn{2}{|c|}{ In PTA $(n=10)$} & \multicolumn{2}{|c|}{ Oriented $(n=10)$} & \multicolumn{2}{|c|}{ Control $(n=187)$} \\
\hline & Median & Range & Median & Range & Median & Range \\
\hline $\begin{array}{l}\text { Age (yr) } \\
\text { Educ (yr) } \\
\text { GCS Score } \\
\text { Unresponsiveness (days) } \dagger \\
\text { Injury-Test Interval (days) } \ddagger\end{array}$ & $\begin{array}{r}20 \\
11^{\mathrm{a}} \\
5 \\
28 \\
104\end{array}$ & $\begin{array}{l}(16-57) \\
(8-20) \\
(4-11) \\
(1-75) \\
(46-411)\end{array}$ & $\begin{array}{l}22 \\
12^{b} \\
6 \\
23 \\
88\end{array}$ & $\begin{array}{l}(18-38) \\
(9-16) \\
5-7) \\
(13-72) \\
(28-216)\end{array}$ & $\begin{array}{l}21 \\
14^{a b} \\
= \\
-\end{array}$ & $\begin{array}{l}(18-51) \\
(13-17) \\
= \\
-\end{array}$ \\
\hline
\end{tabular}

${ }^{*}$ PTA = posttraumatic amnesia; Educ = education; GCS = Glasgow Coma Scale, which has a range of 3 (no eye opening, no motor, or verb response) to 15 (normal level), was recorded on admission.

tDuration of unresponsiveness was estimated from the number of days during which the patient was unable to obey simple commands. Serial GCS scores were obtained from the referring Comprehensive Trauma Center hospital.

\pm Number of days from date of injury to administration of remote memory test of television programs.

$\mathrm{A}_{\mathrm{G}}$ roups with the same superscript letter differed significantly $(\mathrm{p}<0.05)$. 
the test on each of three consecutive days. The correct title and three plausible, but fictitious titles were presented in a randomised vertical arrangement on index cards while the examiner read them aloud. The patient repeated the titles as they were read to verify registration of the information and comprehension of the instructions. Control subjects completed a written, multiple-choice form of the test.

\section{Results}

The mean proportion of correct recognition memory is plotted against the time period of the programmes in fig 1 . Control subjects obtained scores consistently above the scores of the closed head injury groups across all time periods. It can also be seen that the recognition memory of oriented head injured patients no longer in PTA surpassed the performance of the patients in PTA on four of the time periods, whereas both closed head injury groups declined to a chance level on the 1977-1979 programmes. In contrast to the parallel variation in recognition memory across time periods shown for the oriented closed head injury patients and control subjects, there is a relatively flat function for the disoriented group.

A multivariate analysis of variance ${ }^{21}$ performed on the proportions of correct responses disclosed a highly significant overall difference across the three groups (Wilks lambda likelihood ratio $=0.735, \mathrm{~F}=$ 6.64, $\mathrm{p}<0.00001$ ). Univariate $\mathrm{F}$ tests (table 2) confirmed that the retention by control subjects surpassed the performance of discriented closed head injury patients for each time period. As shown in fig 1 , control subjects also obtained higher test scores than the oriented $\mathrm{CHI}$ group on all except the earliest (1968-1971) time periods (table 2). The trend of more accurate remote memory in oriented closed head injury patients as compared to the patients in PTA was not significant (table 2).

To test the overall effect of the time period of the television programmes on remote memory scores and the interaction of time period with the effect of groups, an analysis of variance with repeated measures was performed in which the group (head injury during PTA, head injury after PTA, control subjects) was the between factor and the time period

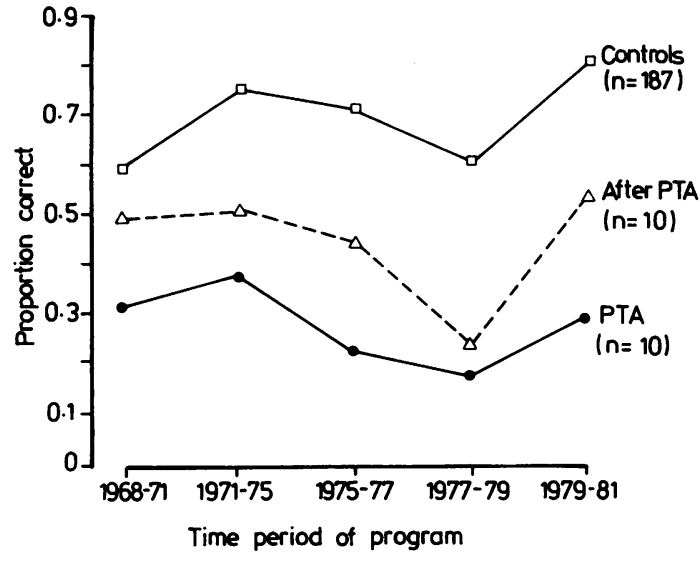

Fig 1 Mean proportion of correct recognition of television program titles plotted across the time period of broadcast for oriented (after PTA) and amnesic (PTA) head injured patients and control subjects.

was the within factor. This analysis confirmed highly significant effects for group $(F=28 \cdot 81, p<$ $0.00001)$, time period $(F=7.98, p<0.00001)$, and a significant interaction of group with time period $(F$ $=2.29, \mathrm{p}<0.03)$. To investigate the sources of this interaction, a trend analysis was performed on the remote memory scores of each group in addition to an overall analysis of the total sample. Moreover, the postulation ${ }^{2}$ that the dissolution of memory is inversely related to the recency of the event would imply the presence of a linear component in the test scores of the head injured groups across the five time periods.

A trend analysis of the remote memory scores of all participants $(n=207)$ across the five time periods disclosed significant overall effects for the quadratic $(\mathrm{F}=7.35, \mathrm{p}<0.008)$ and cubic $(\mathrm{F}=$ $26 \cdot 0, p<0 \cdot 00001)$ components. Consistent with the apparent group differences in their temporal gradients (fig 1), the interaction of groups with trend was significant for the linear $(F=3.23, p<0.05)$ and quadratic components $(F=3.37, p<0.05)$. As reflected by the relatively flat temporal gradient for

Table 2 Univariate contrasts of the proportion of correct recognition memory for items at each time period $\dagger$

\begin{tabular}{lllllll}
\hline Contrast & \multicolumn{2}{l}{ Univariate $F$ values } \\
\cline { 2 - 7 } & $1968-71$ & $1972-75$ & $1975-77$ & $1977-79$ & $1979-81$ & Overall $T^{2 *}$ \\
\hline PTA vs Control & $5 \cdot 82 \dagger$ & $21 \cdot 4 \S$ & $38 \cdot 28$ & $13 \cdot 9 \ddagger$ & $29 \cdot 2 \S$ & $10 \cdot 1 \S$ \\
Oriented vs Control & $0 \cdot 8$ & $5 \cdot 8 \dagger$ & $14 \cdot 2 \ddagger$ & $15 \cdot 01 \S$ & $9 \cdot 5 \ddagger$ & $4.7 \ddagger$ \\
PTA vs Oriented & $1 \cdot 2$ & $1 \cdot 2$ & $2 \cdot 6$ & $3 \cdot 1$ & $0 \cdot 1$ & $1 \cdot 2$ \\
\hline
\end{tabular}

${ }^{*}$ Hotellings $\mathrm{T}^{2}$ is a measure of the composite group difference across the five time periods.

$\nmid \mathrm{p}<0.05$, $\neq \mathrm{p}<0.01$, $\mathrm{p}<0.001$. 
the disoriented group, there was no significant trend in their scores. The gradient of the oriented closed head injury patients (fig 1 ) had significant quadratic $(\mathrm{F}=5 \cdot 10, \mathrm{p}<0.05)$ and cubic $(\mathrm{F}=10.96, \mathrm{p}<$ 0.01) components. Analysis of the control group data, which showed optimal retention for the most recent information, revealed significant effects for the linear $(\mathrm{F}=24.5, \mathrm{p}<0.0001)$ and cubic $(\mathrm{F}=$ $120 \cdot 8, \mathrm{p}<0.0001)$ components.

Table 1 shows a nonsignificant trend for a longer interval between the date of injury and administration of the remote test in the PTA group as compared to the head injured patients who were oriented by the time of the study. The Spearman rank order correlation coefficient between the injury test interval and the total score on the television programme test was $-0.53(p<0.008)$ for the combined groups $(n=20)$, that is, the interval since injury was inversely related to remote memory performance. There was no impressive relationship between the injury-test interval and test score in the PTA group $\left(r_{s}=-0 \cdot 28\right)$, but these variables were correlated in the oriented group $\left(r_{s}=-0.71, p<\right.$ $0 \cdot 02$ ).

\section{Discussion of study I}

Clinical observations and interviews have shown that permanent, complete memory loss for events antedating a non-missile head injury rarely extends retrogradedly to more than two days before the injury. ${ }^{356}$ However, Williams and Zangwill ${ }^{7}$ described a series of closed head injury patients in whom partial, albeit permanent, retrograde memory loss was common for events which occurred more than a week before injury. Although in 1935 Russell $^{5}$ first reported a shrinkage in the interval of RA in those head injured patients who could not initially recall life events which preceded their injury by more than a year, he concluded that the retrograde extent of residual amnesia was generally brief.

Our findings for episodic memories of no personal salience in patients recovering from moderate or severe non-missile head injury indicate that (1) partial RA can extend to memories acquired a decade or more before injury; (2) partial RA can be demonstrated even after head injured patients become normally oriented to time, place, person and circumstances; (3) there is no selective sparing of the oldest, personally nonsalient memories after severe head injury, at least when a standardised, quantitative test of episodic memory is employed. Although our failure to find a temporal gradient in the recognition memory scores of the PTA group may be attributed to their performance approximating a chance level on two time periods (fig 1), there was no evidence of a linear temporal gradient in the remote memory scores of oriented closed head injury patients. In fact, the only confirmation of a linear component was in the control group who exhibited enhanced retention of the most recent television shows. Further, our finding of a quadratic trend in the remote memory scores of the oriented $\mathrm{CHI}$ group is incompatible with the postulation that the dissolution of memory is directly related to its recency. ${ }^{2}$

Consistent with the results in our control group, optimal retention of the most recent information has been previously reported in studies of remote memory in normal elderly subjects ${ }^{16}$ and younger adults. ${ }^{22}$ The relative difficulty of retention for the 1977-1979 programmes in all three groups may reflect idiosyncrasies of the items for this period. Although items for 1968-1979 were derived from a pool which showed only minor deviation from a linear trend in normal subjects, ${ }^{22}$ we employed an abbreviated form of the test because of reduced capacity for sustained attention in our patients. These features of the television programme test may account for the presence of a cubic trend in the remote memory scores of both the oriented closed head injury and control groups.

Matching the PTA and oriented groups on the severity of initial injury (that is GCS score) was of primary concern and we anticipated variation in the injury-test interval. Although these groups did not significantly differ in the interval since injury, it was inversely related to their accuracy of remote memory particularly in the patients whose PTA had resolved. The modest correlation in the PTA group may reflect their more uniformly impaired test scores as compared to wider variation in the remote memory of the oriented group. While this incidental finding warrants closer study in a larger series, it suggests that a long period of disorientation, confusion and gross anterograde amnesia may be related to a residual decrement in remote memory even after clearing of PTA.

\section{STUDY II}

The clinical observation that the oldest memories are more resistant to disruption after head injury ${ }^{2}$ may reflect the emphasis on overlearned, personally salient information when clinicians examine the retention of events from the remote past (for example place of birth). Secondly, collateral information from a relative or friend is necessary to verify the accuracy of the patient's recall. In view of the disparity between the results of Study I and the wide acceptance of Ribot's formulation, we investigated remote memory for autobiographic information by 
using a structured interview of major life events. We postulated that reminiscence of important childhood experiences facilitates their retention as semantic knowledge rather than as episodic events and thereby produces a temporal gradient in RA.

\section{Patients and methods}

\section{Patients}

The head injured patients were selected from the same inpatient rehabilitation service and evaluated for PTA according to the same criteria that were used in Study I. In addition, a relative or close friend had to be available to provide biographical information and the patient's preinjury history had to contain the events included in the assessment. Of the 20 closed head injury patients included in Study I, eight also participated in Study II. Demographic features, the initial GCS score, and the duration of coma are summarised for the PTA $(n=6)$ and oriented groups $(n=17)$ in table 3 . There were no differences between the groups on these variables. Based on CT and surgical findings, the PTA group $(n=6)$ included one patient with a left hemisphere mass lesion, three patients with right hemisphere mass lesions, and two cases of bilateral injury. Of the 17 patients tested after PTA termination, 13 had hemispheric mass lesions (five left, two right, six bilateral) and four had diffuse injuries.

\section{Procedure}

The autobiographical inventory, which we developed primarily for use with young head injured adults, was divided into four developmental periods spanning primary school, intermediate or junior high school, high school, and post-secondary school or young adulthood. After obtaining the correct information from a collateral source (usually a

Table 3 Demographic and clinical features of head injured patients in PTA and oriented patients who were tested for autobiographic memory in Study II*

\begin{tabular}{|c|c|c|c|c|}
\hline & \multicolumn{2}{|c|}{ In PTA $(n=6)$} & \multicolumn{2}{|c|}{ Oriented $(n=17)$} \\
\hline & Median & Range & Median & Range \\
\hline $\begin{array}{l}\text { Age (yr) } \\
\text { Education (yr) } \\
\text { GCS Score } \\
\text { Unresponsiveness }\end{array}$ & $\begin{array}{r}27 \cdot 5 \\
12 \cdot 8 \\
7 \cdot 0 \\
19 \cdot 8\end{array}$ & $\begin{array}{l}(24-30) \\
(12-15) \\
(3-12) \\
(10-82)\end{array}$ & $\begin{array}{r}26 \cdot 3 \\
12 \cdot 0 \\
6 \cdot 0 \\
23 \cdot 6\end{array}$ & $\begin{array}{l}(18-40) \\
\left(\begin{array}{l}9-20) \\
4-9) \\
1-72)\end{array}\right.\end{array}$ \\
\hline $\begin{array}{l}\text { Injury-Test Interval } \\
\text { (days) } \ddagger\end{array}$ & $71 \cdot 5$ & $(54-160)$ & $109 \cdot 0$ & $(28-491)$ \\
\hline
\end{tabular}

*PTA = posttraumatic amnesia; GCS = Glasgow Coma Scale, which has a range of 3 (no eye opening, no motor, or verbal response) to 15 (normal level) was recorded on admission.

†Duration of unresponsiveness was estimated from the number of days during which the patient was unable to obey simple commands. Serial GCS scores were obtained from the Trauma Center hospital.

$\ddagger$ Number of days from date of injury to assessment of autobiographical memory. relative), the patient was tested for recall of autobiographic material by using a brief structured interview. There were eight questions, including two questions for each time period. Half of the questions consisted of two parts. The questions pertaining to the primary school years were: "What was the name of your elementary school?" and "In regard to the first time you can remember your family moving, how old were you (age or grade in school) and where did you move to (name of town or street)?" In the event that the patient did not move during the primary school years, recall of his or her address at that time was tested. The questions for the intermediate or junior high school period were: "What was the name of your junior high school?" and "With whom did you have your first date (first name)?" and "How old were you then (age or grade)?" The questions corresponding to the high school period included: "What was the name of your high school and when did you graduate or drop out (year)?" and "On what type of car did you learn to drive (make and year)?" The questions corresponding to the post-secondary schoolyoung adulthood period were: "What was the address of your first residence after leaving home?" and "On what date did you marry your wife (husband)?" The date at which the patient began his or her first long term heterosexual relationship during this period was substituted for unmarried patients. If the patient failed to respond to a question, a prompt consisting of the first two letters of the correct answer was given. Half-credit was awarded for correct answers which required a prompt.

\section{Results}

The proportion of correct responses was scored for each developmental period and plotted in fig 2 for the PTA and oriented head injured groups. Figure 2 shows a trend of more accurate recall of autobiographical data by head injured patients who had regained orientation as compared to the PTA group. In contrast to the relatively slight variation in accuracy of recall by the oriented patients across time periods, fig 2 depicts a temporal gradient for the recall by patients who were still in PTA when tested. Recall accuracy decreased monotonically from relatively preserved retention of information from childhood to retrograde memory loss of personal data during late adolescence and young adulthood.

A multivariate analysis of variance ${ }^{21}$ performed on the proportions of correct responses confirmed the impression of more accurate recall of autobiographical data by oriented $\mathrm{CHI}$ patients as compared to the patients in PTA (table 4). As summarised in table 4 , head injured patients who were examined during PTA exhibited impoverished recall of autobiographical information from the three most recent time periods. In contrast, the trend of more accurate recall by oriented closed head injury patients than the group in PTA did not approach significance for autobiographical data sampled from 


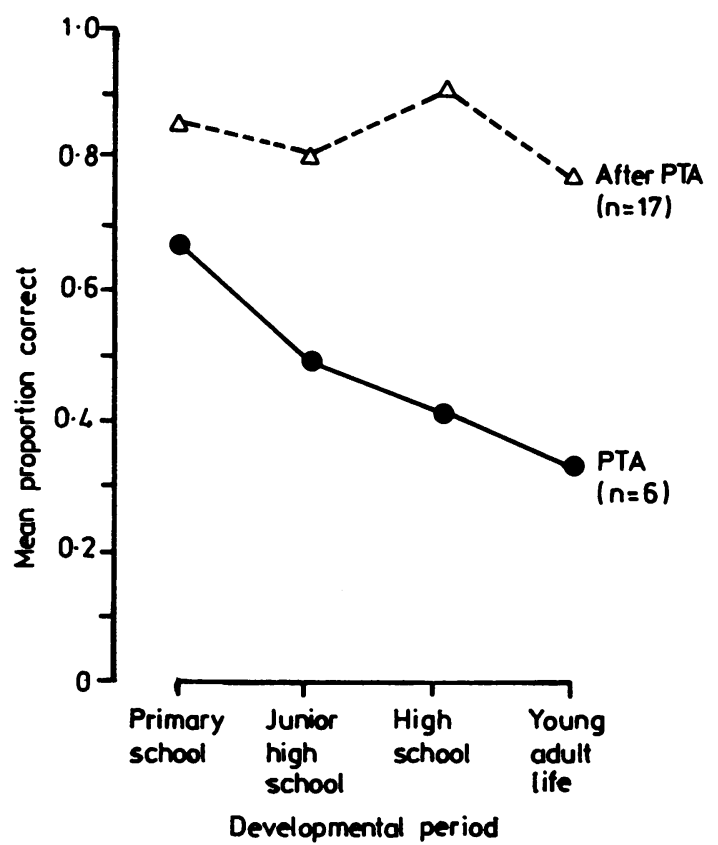

Fig 2 Mean proportion of correct recall of autobiographical events plotted across developmental periods for head injured patients in PTA $(n=6)$ and after PTA $(n=17)$.

primary school years. To compare the differential recall of autobiographical information derived from four developmental periods in the oriented closed head injury patients vs the head injury group in PTA, an analysis of variance with repeated measures was performed. The results confirmed a highly significant effect for group $(F=14.93, p<0.001)$, and developmental period $(F=5.00, p<0.004)$. Consistent with the impression in fig 2 for wider divergence in autobiographical memory between the oriented and disoriented/confused closed head injury groups for the most recent developmental periods, there was a significant interaction between the group and developmental period $(F=2 \cdot 88, p<$ 0.05 ). To elucidate the sources of this interaction and test the presence of a linear component in the autobiographical memory scores across time periods, a trend analysis was completed.
The trend analysis confirmed the impression of a significant overall linear component $(\mathrm{F}=12 \cdot 71, \mathrm{p}<$ $0.002)$ for the combined groups $(n=23)$. Consistent with the contrasting temporal gradients of the PTA and oriented groups (fig 2), there was a significant interaction of groups with the linear component of the trend analysis $(F=6.37, p<0.02)$. This analysis was then repeated for the PTA and oriented groups separately. The linear decline in recall with increasing recency of events by patients in PTA was significant $(F=6.26, p<0.05)$, whereas no other component in their autobiographical memory scores reached significance. A similar analysis disclosed a cubic trend which only approached significance $(p<0 \cdot 10)$ in the oriented group.

Table 3 indicates a nonsignificant trend for a longer interval between injury and testing in the oriented group. There was no correlation between time since injury and retention of autobiographical information in the combined groups $\left(n=23, r_{s}=\right.$ $-0 \cdot 06)$ and in the oriented group $\left(r_{s}=0 \cdot 16\right)$, but there was an inverse relationship in the PTA group which approached significance $\left(r_{s}=-0.71, p<\right.$ 0.06).

\section{Discussion of Study II and general discussion}

Clinical interviewing of consecutive closed head injured patients after resolution of PTA has established that more than half report RA for events up to about a minute preinjury, whereas retrograde extension of complete amnesia to more than two days is rare. ${ }^{56}$ Brief RA confined to immediate preinjury events has been attributed to disruption of memory consolidation, that is, encoding and storage, whereas impaired retention of events from the distant past has been interpreted as retrieval failure. ${ }^{4-61423}$ Consistent with this interpretation of extensive RA as faulty retrieval, Sanders and Warrington ${ }^{14}$ postulated that proactive interference from prior learning uniformly impairs recall of events from various time periods. Accordingly, some instances of confabulation by head injured patients during PTA 5620 could represent an earlier experience being recalled out of context. The phenomenon of "shrinking" RA, that is, a reduction in the ini-

Table 4 Univariate contrasts of the proportions of correct recall of autobiographical data by the PTA and oriented head injury groups

\begin{tabular}{llll}
\hline & $\begin{array}{l}\text { Univarient F values } \\
\text { PTA vs oriented }\end{array}$ \\
\hline $\begin{array}{l}\text { Primary School } \\
2 \cdot 28\end{array}$ & $\begin{array}{l}\text { Intermediate/Junior High School } \\
8 \cdot 16 \dagger\end{array}$ & $\begin{array}{l}\text { High School } \\
37 \cdot 47 \ddagger\end{array}$ & $\begin{array}{l}\text { Young Adult } \\
10 \cdot 01 \dagger\end{array}$ \\
\hline
\end{tabular}

${ }^{*} p<0.01, \neq p<0.005, \neq p<0.0001$. 
tially long time period for which memory of past events is lost, has also been viewed as a reversible retrieval deficit. ${ }^{46}$ Although most investigators have considered prolonged RA to be an infrequent feature of closed head injury after restoration of orientation and memory of ongoing events, partial disruption of remote memories has been reported after PTA resolves.?

Our results indicate that during the subacute stage of recovery from moderate or severe non-missile head injury, patients exhibit at least partial amnesia for events which occurred during the preceding decade. We demonstrated impaired remote memory for both events in the public domain (for which we assumed at least incidental exposure) and life events which are salient for most persons. Consequently, inadequate initial acquisition or lack of exposure cannot account for the findings in both Studies I and II. Secondly, we found that remote memory for life events was impaired in head injured patients who were still exhibiting disorientation and marked anterograde amnesia as compared to patients with head injuries of comparable severity who had emerged from PTA. Previous studies ${ }^{4-6}$ have documented an association of prolonged, residual RA with relatively long PTA suggesting that "the retrieval process depends on the same system that is necessary for the laying down of new memories" (p. 542). ${ }^{4}$

Investigators have debated the presence of a temporal gradient in patients with other aetiologies of amnesic disorder who exhibit impaired remote memory. ${ }^{10-16}$ Although our results for retention of titles of television shows provide no support for Ribot's postulation that the strength of memories increases over time, we found relative preservation of the earliest autobiographical memories during PTA. Head injured patients who had emerged from PTA, however, accurately recalled at least $80 \%$ of life events queried for each developmental period while exhibiting no impressive trend in relation to the age at which the memories were acquired. Although the accuracy of recall of autobiographical information was relatively uniform across the intervals in patients tested after resolution of PTA, the issue of equivalence of item difficulty for different developmental periods requires further study.

How can we explain the relative preservation of early autobiographical memories during PTA despite impaired retention of television programme titles from corresponding periods? Ribot ${ }^{2}$ postulated that repeated retrieval of remote memories increases their resistance to decay or disruption. Hence, we suggest that repeated reminiscence of early personal events incorporated these memories into a semantic structure which became relatively invulnerable to RA. In contrast, we suggest that this reminiscence effect was diminished for more recent life events and negligible for titles of previous television programmes.

The temporal gradient in the remote memory of Korsakoff patients ${ }^{y} 1112$ and in cases tested after a long interval following bilateral hippocampal excisions $^{9}$ or diencephalic injury ${ }^{10}$ may reflect the cumulative effects of marked anterograde amnesia, that is inadequate acquisition of the information. Individual differences in the progression of anterograde amnesia in alcoholic Korsakoff patients might account for the observed variation in the accuracy of their later retention of public events and faces of prominent persons and the inconsistent finding of a temporal gradient in their remote memory. ${ }^{1423}$ In fact, Mair, Warrington and Weiskrantz ${ }^{23}$ showed that no temporal gradient was present in the remote memory of two necropsy-confirmed cases of alcoholic Korsakoff syndrome. Both patients exhibited impaired recall and recognition for public events and well known faces which extended over at least a 25 year period. While the necessary and sufficient conditions for the presence of a temporal gradient in the remote memory of alcoholic Korsakoff patients may be disputed, it is clear that the onset of their amnesic disorder is difficult to define precisely. In contrast, our investigation of RA during the early stages of recovery after head injury permits precise specification of the onset of amnesia.

Apart from the theoretical implications of our findings for the neuropsychology of memory, we postulate that partial retrograde memory disturbance after head injury can adversely affect resumption of complex activities which stress retention of previously acquired, but not overlearned information. Elucidation of the clinical course of residual RA following head injury awaits a longitudinal extension of this study.

This research was supported by grants NS 21889 , Javits Neuroscience Investigator Award, NS 07377, Center for the Study of Nervous System Injury, Moody Foundation Grants 80-233, 82-47 and 82241, and Contract 9-2314, Comprehensive Central Nervous System Injury Trauma Center. We are grateful to Dr AL Benton for his critique of the article, to Beverly White for manuscript preparation and to Dr LR Squire for providing the television program test.

\section{References}

' Levin HS, Peters BH, Hulkonen DA. Early concepts of anterograde and retrograde amnesia. Cortex 1983; 19:427-40. 
${ }^{2}$ Ribot T. Diseases of Memory: An Essary in the Positive Psychology. New York: Appleton, 1882.

${ }^{3}$ Russell WR. Cerebral involvement in head injury. Brain 1932;55:549-603.

${ }^{4}$ Benson DF, Geschwind N. Shrinking retrograde amnesia. J Neurol Neurosurg Psychiatry 1967; 30:539-44.

${ }^{5}$ Russell WR. Amnesia following head injuries. Lancet 1935;2:762-3.

- Russell WR, Nathan PW. Traumatic amnesia. Brain 1946;69: 183-7.

7 Williams M, Zangwill OL. Memory defects after head injury. J Neurol Neurosurg Psychiatry 1952;15:54-58.

${ }^{8}$ Squire LR, Slater PC, Chace PM. Retrograde amnesia: Temporal gradient in very long-term memory following electroconvulsive therapy. Science 1975;187: 77-9.

${ }^{4}$ Marslen-Wilson WD, Teuber H-L. Memory for remote events in anterograde amnesia: Recognition of public figures from news-photographs. Neuropsychologia 1975; 13:353-64.

${ }^{10}$ Squire LR, Slater PC. Anterograde and retrograde memory impairment in chronic amnesia. Neuropsychologia 1978; 16:313-22.

" Albert MS, Butters N, Levin J. Temporal gradients in the retrograde amnesia of patients with alcoholic Korsakoff's disease. Arch Neurol 1979;36:211-6.

${ }^{12}$ Seltzer B, Benson DF. The temporal pattern of retrograde amnesia in Korsakoff's disease. Neurology (Minneap) 1974;24:527-30.

${ }^{13}$ Cermak LS, O'Connor $M$. The anterograde and retrograde retrieval ability of a patient with amnesia due to encephalitis. Neuropsychologia 1983;21:21334.

14 Sanders HI, Warrington EK. Memory for remote events in amnesic patients. Brain 1971;94:661-8.

15 Squire LR. Remote memory as affected by aging. Neuropsychologia 1974;12:429-35.

16 Warrington EK, Sanders HI. The fate of old memories. Q J Exp Psychol 1971;23:432-42.

17 Levin HS, O' Donnell VM, Grossman RG. The Galveston orientation and amnesia test: A practical scale to assess cognition after head injury.J Nerv Ment Dis 1979; 167:675-84.

${ }^{18}$ Teasdale G, Jennett B. Assessment of coma and impaired consciousness: A practical scale. Lancet 1974;2:81-4.

${ }^{19}$ Squire LR, Slater PC. Forgetting in very long-term memory as assessed by an improved questionnaire technique. J Exp Psychol: Human Learning and Memory 1975; 1:50-4.

${ }^{20}$ Levin HS, Benton AL, Grossman RG. Neurobehavioral Consequences of Closed Head Injury. New York and Oxford: Oxford University Press, 1982.

${ }^{21}$ Dixon WJ. BMDP Statistical Software. Berkeley, California: University of California Press, 1981.

22 Squire LR, Fox MM. Assessment of remote memory: Validation of the television test by repeated testing during a 7-year period. Behav Res Meth Instr 1980;12:583-6.

${ }^{23}$ Mair WGP, Warrington EK, Weiskrantz L. Memory disorder in Korsakoff's psychosis. A neuropathological and neuropsychological investigation of two cases. Brain 1979;102:749-83. 